EPJ Web of Conferences 66, 09011 (2014)

DOI: 10.1051/epjconf/ 20146609011

(C) Owned by the authors, published by EDP Sciences, 2014

\title{
Low mass dielectrons radiated off cold nuclear matter measured with HADES
}

M. Lorenz ${ }^{7,18, a}$ G. Agakishiev6 ${ }^{6}$ C. Behnke ${ }^{7}$, D. Belver ${ }^{16}$, A. Belyaev ${ }^{6}$, J.C. Berger-Chen ${ }^{8}$, A. Blanco ${ }^{1}$, C. Blume ${ }^{7}$, M. Böhmer ${ }^{9}$, P. Cabanelas ${ }^{16}$, S. Chernenko ${ }^{6}$, C. Dritsa ${ }^{10}$, A. Dybczak ${ }^{2}$, E. Epple ${ }^{8}$, L. Fabbietti ${ }^{8,9}$, O. Fateev ${ }^{6}$, P. Fonte ${ }^{1,17}$, J. Friese ${ }^{9}$, I. Fröhlich ${ }^{7}$, T. Galatyuk ${ }^{4,18}$, J. A. Garzón ${ }^{16}$, K. Gill ${ }^{7}$, M. Golubeva ${ }^{11}$, D. González-Díaz ${ }^{4}$, F. Guber ${ }^{11}$, M. Gumberidze ${ }^{14}$, S. Harabasz ${ }^{4}$, T. Hennino ${ }^{14}$, C. Höhne ${ }^{10}$, R. Holzmann ${ }^{3}$, P. Huck ${ }^{9}$, A. lerusalimov ${ }^{6}$, A. Ivashkin ${ }^{11}$, M. Jurkovic ${ }^{9}$, B. Kämpfer ${ }^{5,19}$, T. Karavicheva ${ }^{11}$, I. Koenig ${ }^{3}$, W. Koenig ${ }^{3}$, B. W. Kolb ${ }^{3}$, G. Korcyl' G. Kornakov ${ }^{16}$, R. Kotte ${ }^{5}$, A. Krása ${ }^{15}$, E. Krebs ${ }^{7}$, F. Krizek ${ }^{15}$, H. Kuc ${ }^{2,14}$, A. Kugler ${ }^{15}$, A. Kurepin ${ }^{11}$,

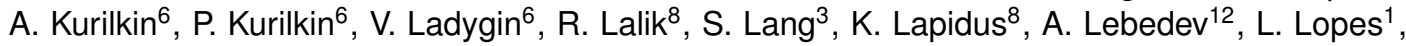
L. Maier ${ }^{9}$, A. Mangiarotti ${ }^{1}$, J. Markert ${ }^{7}$, V. Metag ${ }^{10}$, J. Michel ${ }^{7}$, C. Müntz ${ }^{7}$, R. Münzer ${ }^{8}$, L. Naumann ${ }^{5}$, M. Palka ${ }^{2}$, Y. Parpottas ${ }^{13,20}$, V. Pechenov ${ }^{3}$, O. Pechenova ${ }^{7}$, J. Pietraszko ${ }^{7}$, W. Przygoda ${ }^{2}$, B. Ramstein ${ }^{14}$, L. Rehnisch ${ }^{7}$, A. Reshetin ${ }^{11}$, A. Rustamov ${ }^{7}$, A. Sadovsky ${ }^{11}$, P. Salabura ${ }^{2}$, T. Scheib ${ }^{7}$, H. Schuldes ${ }^{7}$, J. Siebenson ${ }^{8}$, Yu.G. Sobolev ${ }^{15}$, S. Spataro ${ }^{21}$, H. Ströbele ${ }^{7}$, J. Stroth ${ }^{7,3}$, P Strzempek ${ }^{2}$, C. Sturm ${ }^{3}$, O. Svoboda ${ }^{15}$, A. Tarantola ${ }^{7}$, K. Teilab ${ }^{7}$, P. Tlusty ${ }^{15}$, M. Traxler ${ }^{3}$, H. Tsertos ${ }^{13}$, T. Vasiliev ${ }^{6}$, V. Wagner ${ }^{15}$, M. Weber ${ }^{9}$, C. Wendisch ${ }^{5,19}$, J. Wüstenfeld ${ }^{5}$, S. Yurevich ${ }^{3}$, Y. Zanevsky ${ }^{6}$

${ }^{1}$ LIP-Laboratório de Instrumentação e Física Experimental de Partículas , 3004-516 Coimbra, Portugal

${ }^{2}$ Smoluchowski Institute of Physics, Jagiellonian University of Cracow, 30-059 Kraków, Poland

${ }^{3}$ GSI Helmholtzzentrum für Schwerionenforschung GmbH, 64291 Darmstadt, Germany

${ }^{4}$ Technische Universität Darmstadt, 64289 Darmstadt, Germany

${ }^{5}$ Institut für Strahlenphysik, Helmholtz-Zentrum Dresden-Rossendorf, 01314 Dresden, Germany

${ }^{6}$ Joint Institute of Nuclear Research, 141980 Dubna, Russia

${ }^{7}$ Institut für Kernphysik, Goethe-Universität, 60438 Frankfurt, Germany

${ }^{8}$ Excellence Cluster 'Origin and Structure of the Universe', 85748 Garching, Germany

${ }^{9}$ Physik Department E12, Technische Universität München, 85748 Garching, Germany

${ }^{10}$ II.Physikalisches Institut, Justus Liebig Universität Giessen, 35392 Giessen, Germany

${ }^{11}$ Institute for Nuclear Research, Russian Academy of Science, 117312 Moscow, Russia

${ }^{12}$ Institute of Theoretical and Experimental Physics, 117218 Moscow, Russia

${ }^{13}$ Department of Physics, University of Cyprus, 1678 Nicosia, Cyprus

${ }^{14}$ Institut de Physique Nucléaire, CNRS/IN2P3 - Université Paris Sud, F-91406 Orsay Cedex, France

${ }^{15}$ Nuclear Physics Institute, Academy of Sciences of Czech Republic, 25068 Rez, Czech Republic

${ }^{16}$ LabCAF. F. Física, Univ. de Santiago de Compostela, 15706 Santiago de Compostela, Spain

${ }^{17}$ Also at ISEC Coimbra, Coimbra, Portugal

${ }^{18} \mathrm{Also}$ at ExtreMe Matter Institute EMMI, 64291 Darmstadt, Germany

${ }^{19}$ Also at Technische Universität Dresden, 01062 Dresden, Germany

${ }^{20}$ Also at Frederick University, 1036 Nicosia, Cyprus

${ }^{21}$ Also at Dipartimento di Fisica Generale and INFN, Università di Torino, 10125 Torino, Italy

a e-mail: m.lorenz@gsi.de

This is an Open Access article distributed under the terms of the Creative Commons Attribution License 2.0, which permits unrestricted use, distribution, and reproduction in any medium, provided the original work is properly cited. 


\begin{abstract}
The High Acceptance DiElectron Spectrometer HADES [1] is installed at the Helmholtzzentrum für Schwerionenforschung (GSI) accelerator facility in Darmstadt. It investigates dielectron emission and strangeness production in the 1-3 AGeV regime. A recent experiment series focusses on medium-modifications of light vector mesons in cold nuclear matter. In two runs, $\mathrm{p}+\mathrm{p}$ and $\mathrm{p}+\mathrm{Nb}$ reactions were investigated at $3.5 \mathrm{GeV}$ beam energy; about $9 \cdot 10^{9}$ events have been registered. In contrast to other experiments the high acceptance of the HADES allows for a detailed analysis of electron pairs with low momenta relative to nuclear matter, where modifications of the spectral functions of vector mesons are predicted to be most prominent. Comparing these low momentum electron pairs to the reference measurement in the elementary $p+p$ reaction, we find in fact a strong modification of the spectral distribution in the whole vector meson region.
\end{abstract}

\title{
1 Introduction
}

The prospect to relate hadron properties inside a strongly interacting medium to chiral symmetry restoration has motivated plenty of activities, both in theoretical and experimental physics. The spontaneous breaking of chiral symmetry leads to non vanishing values of chiral condensates, which are connected to the dynamical generation of hadron masses. Focussing mainly on vector mesons, various models predict relatively strong changes of particle masses and/or widths already at normal nuclear matter density $\rho_{0}$ [2-8]. However, a consistent picture of in-medium hadron properties has not yet emerged and more experimental input is needed. Moreover, these calculations are restricted to particles at rest or small relative momenta to the surrounding medium, a region which is extremely challenging to be accessed in the experiments.

Experimentally, the in-medium properties can be studied in heavy-ion collisions (probing hot and dense hadronic matter) $[9,10]$ or in proton-, pion- or photon- induced reactions on nuclei (cold nuclear matter) [11-14]; while $\mathrm{e}^{+} \mathrm{e}^{-}$pair spectroscopy is an ideal tool for such studies due to the fact that electrons and positrons interact only electromagnetically and their kinematics stay essentially undistorted while propagating through the surrounding nuclear matter.

\section{Experimental data and discussion}

In this paper we highlight our findings on inclusive $\mathrm{e}^{+} \mathrm{e}^{-}$pair production in proton induced reactions at $\mathrm{E}_{\text {kin }}=3.5 \mathrm{GeV}$ on a hydrogen and on a $\mathrm{Nb}$ target, representing the first high statistics measurement with small $\mathrm{e}^{+} \mathrm{e}^{-}$pairs relative momenta to the medium $\left(P_{e e}<0.8 \mathrm{GeV} / \mathrm{c}\right)$ published in $[16,17]$. By comparing the two data sets the presented results are sensitive to line shape modifications and nuclear suppression.

However, as several broad overlapping contributions add up to the total dielectron yield it is important to develop a good understanding of the different contributions and to get as many additional experimental constraints as possible. Therefore we start by discussing the elementary reference process first. For the $p+p$ data a dielectron cocktail was generated using an adapted version of the event generator PYTHIA, see [16] for details. There are four distinguished mass regions, namely: $M_{e e}\left[\mathrm{GeV} / \mathrm{c}^{2}\right]<0.15$ (dominated by neutral pion decays), $0.15<M_{e e}\left[\mathrm{GeV} / \mathrm{c}^{2}\right]<0.47$ ( $\eta$ decay dominated), $0.47<M_{e e}\left[\mathrm{GeV} / \mathrm{c}^{2}\right]<0.7$ (dominated by $\rho$ decays and Dalitz decays of baryonic resonances, as well as Dalitz decays of $\omega$ mesons) and $0.7<M_{e e}\left[\mathrm{GeV} / \mathrm{c}^{2}\right]$ (vector meson dominated) as can be seen from the cocktail in Fig. 1. Meanwhile, for the $\mathrm{p}+\mathrm{Nb}$ data set the $\eta$ contribution could be constrained making use of the double photon conversion within the detector [18], the corresponding signal is displayed in Fig. 2. Already in elementary collisions the present theoretical modeling of 


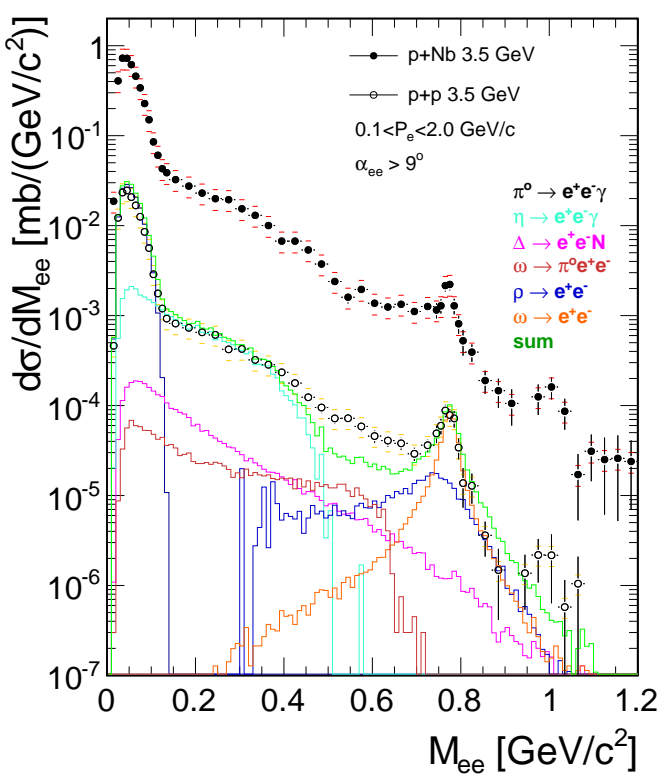

Figure 1. Comparison of dielectron cross sections as a function of the invariant mass measured in $\mathrm{p}+\mathrm{p}$ and $\mathrm{p}+\mathrm{Nb}$ collisions. The $\mathrm{p}+\mathrm{Nb}$ data are displayed with full circles and red horizontal lines indicating the systematical errors, while the $\mathrm{p}+\mathrm{p}$ data are displayed with open circles and yellow horizontal lines. For the $\mathrm{p}+\mathrm{p}$ data a dilepton cocktail is displayed in addition.

short lived contributions from $\rho$ and $\Delta$ decays results in an underestimation of the dielectron yield in the mass region from $0.47<M_{e e}\left[\mathrm{GeV} / \mathrm{c}^{2}\right]<0.7$. It turned out that a more satisfactory description of the data can be achieved by increasing the yield of $\rho$-like contributions by introducing a strong coupling between baryonic resonances and $\rho$ mesons $[19,20]$. However, the question arises whether it is still meaningful to distinguish between the different contributions as they are strongly coupled anyhow.

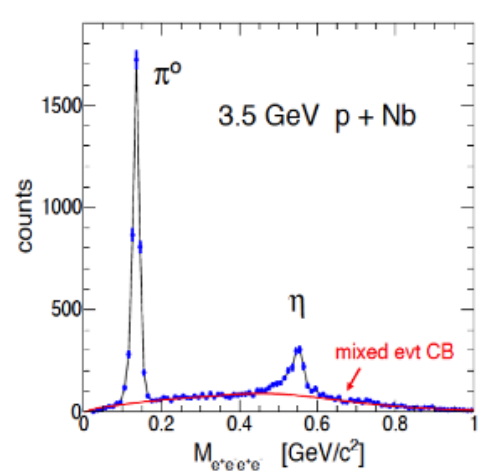

Figure 2. Distribution of the 4-electron invariant mass (dots, error bars are statistical) measured with HADES in the $3.5 \mathrm{GeV}$ p $+\mathrm{Nb}$ reaction. To improve visibility, the data points are connected by a thin curve. The background of uncorrelated lepton combinations obtained from event mixing is shown as well (solid curve).

Comparing the shape of the $\mathrm{p}+\mathrm{p}$ data to the $\mathrm{p}+\mathrm{Nb}$ data in the vector meson region we make the following observations: While the spectral shape in the vector meson region shows no deviation from the reference measurement in $\mathrm{p}+\mathrm{p}$ collisions for $\mathrm{e}^{+} \mathrm{e}^{-}$pairs with momenta larger than $0.8 \mathrm{GeV} / \mathrm{c}$, pairs with smaller momenta exhibit a strong difference in their spectral shape attributed to additional $\rho$ like contributions, see Fig. 3. While this behavior extends to pairs over a wide mass range, the $\omega$ meson signal shows no dependence on momentum. 


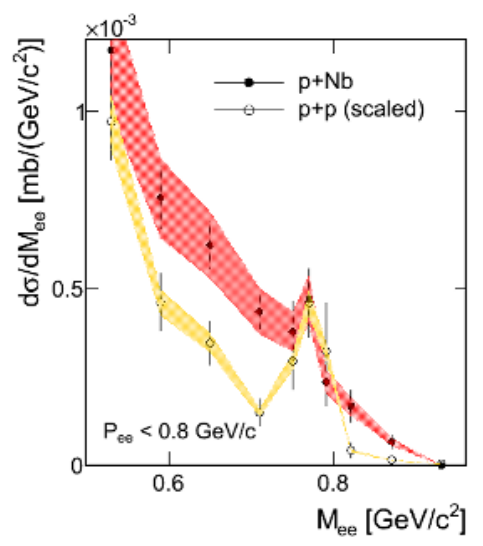

Figure 3. Linear comparison of the invariant mass spectra for $\mathrm{e}^{+} \mathrm{e}^{-}$ pairs in the vector meson mass region and $P_{e e}<0.8 \mathrm{GeV} / \mathrm{c}$ from $\mathrm{p}+\mathrm{p}$ and $\mathrm{p}+\mathrm{Nb}$.

\section{Acknowledgements}

Work supported by BMBF 06FY9100I, 06FY7114, HIC for FAIR, HGS-Hire, EMMI and GSI.

\section{References}

[1] G. Agakishiev et al. (HADES Collaboration), Eur. Phys. J. A 41, 243 (2009).

[2] R. Brockmann and W. Weise, Phys. Lett. B 367, 40 (1996).

[3] G. E. Brown and M. Rho, Phys. Rev. Lett. 66, 2720 (1991).

[4] T. Hatsuda and S. H. Lee, Phys. Rev. C 46, R34 (1992).

[5] V. Bernard and Ulf G. Meissner, Nucl. Phys. A489, 647 (1988).

[6] R. Rapp et al., Nucl. Phys. A617, 472 (1997).

[7] M. Post, S. Leupold and U. Mosel, Nucl. Phys. A 741 81-148 (2004).

[8] S. Leupold, V. Metag, U. Mosel Int. J. Mod. Phys. E19, 147-224 (2010)

[9] A. Uras et al. (NA60 Collaboration), J. Phys. G G38 124180 (2011).

[10] G. Agakishiev et al. (HADES Collaboration), Phys. Rev. C 84 (2011).

[11] R. Nasseripour et al. (CLAS Collaboration) Phys. Rev. Lett. 99, 262302 (2007).

[12] M. Naruki et al., Phys. Rev. Lett. 96, 092301 (2006).

[13] M. Kotulla et al., (CBELSA-TAPS Collaboartion) Phys. Rev. Lett. 100, 192302 (2008).

[14] T. Ishikawa et al. (SPring-8 Collaboration), Phys. Lett. B 608, 215-222 (2005).

[15] A. Polyanskiy et al. (ANKE Collaboration), Phys. Lett. B 695, 74 (2011).

[16] G. Agakishiev et al. (HADES Collaboration), arXiv:1112.3607 [nucl-ex].

[17] G. Agakishiev et al. (HADES Collaboration), Phys. Lett. B 715304 (2012).

[18] G. Agakishiev et al. (HADES Collaboration), arXiv:1305.3118 [nucl-ex].

[19] J. Weil, K. Gallmeister and U. Mosel,PoS BORMIO 053 (2011), arXiv:1105.0314 [nucl-th].

[20] J. Weil, H. van Hees, and U. Mosel, Eur. Phys. J. A 48111 (2012). 